\title{
Spectroscopic Study of Oxygen Sorption and Diffusion in a Membrane Containing a Cobalt Porphyrin Complex
}

\author{
Hiroyuki Nishide, Manshi OHYANAGI, Osamu OKADA, \\ and Eishun TSUCHIDA \\ Department of Polymer Chemistry, Waseda University, \\ Tokyo 160, Japan
}

(Received September 27, 1986)

\begin{abstract}
The kinetics and equilibrium of molecular oxygen sorption in the membrane containing $\left[\alpha, \alpha^{\prime}, \alpha^{\prime \prime}, \alpha^{\prime \prime \prime}\right.$-meso-tetrakis(o-pivalamidophenyl)porphinato $]$ cobalt(II)-1-methylimidazole (CoPIm) complex which forms oxygen adduct rapidly and reversibly are studied spectroscopically. Oxygen sorption in the membrane containing the complex is interpreted in terms of dual mode sorption, whereby the sorbed oxygen exists as two populations: one sorbed to a polymer matrix according to Henry's law and the other to the fixed complex according to the Langmuir isotherm. The diffusion of oxygen in the membrane is found to be Fickian. Apparent oxygen diffusivity increases with an increase in the upstream oxygen pressure, which is in accordance with the dual mode transport model.
\end{abstract}

KEY WORDS Oxygen Sorption / Oxygen Diffusion / Dual Mode Transport

Theory / Cobalt Porphyrin / Poly(butyl methacrylate) /

There has been some interest in gas transports in glassy polymers which behave in accordance with the dual mode transport theory. ${ }^{1-3}$ The dual mode gas transport process has often been investigated for the sorptions of large condensable penetrants in glassy polymers using a gravimetric sorption rate measurement. ${ }^{4}$ But in these investigations plasticization of the glassy polymers occurred gradually during the penetrant-sorption in the polymers due to their non-equilibrium nature. ${ }^{5}$ This produced factors which complicated the analysis of the transport phenomenon using the dual mode transport theory. ${ }^{4}$ Additionally, the nature of a frozen free volume or a microvoid as the Langmuir sorption site is not yet elucidated and there still remains a degree of uncertainty.

The diffusion profile of gases in silicone membrane containing adsorptive fillers has been reported, ${ }^{6}$ but the study was confined to immobilizing adsorption to the fillers and to discussion on the diffusion time lag. Recently the authors succeeded in preparing a rubbery poly(butyl methacrylate) membrane containing $\left[\alpha, \alpha^{\prime}, \alpha^{\prime \prime}, \alpha^{\prime \prime \prime}\right.$-meso-tetrakis $(o$-pivalamidophenylporphinato]cobalt(II)-1-methylimidazole (CoPIm) complex with rapid and reversible oxygen-binding ability even in the solid state, ${ }^{7}$ through which oxygen permeated with high selectivity in comparison to nitrogen. ${ }^{8,9}$ The membrane was transparent and wine red colored and the reversible oxygen-binding to the cobalt complex could be observed in situ with the spectral change in visible region. In this paper, the oxygen sorption kinetics to this membrane were studied by estimating the amount of sorption spectroscopically. The oxygen-binding parameters determined spectroscopically were approximately adequate for analyzing the dual mode transport model. There are some merits for this spectroscopic method of measuring the oxygen sorption in a polymer membrane in comparison with the 
gravimetric method: the measurement is conducted with a small amount of the membrane sample, simply and in a short time, and with high reproducibility.

\section{EXPERIMENTAL}

\section{Materials}

The poly(butyl methacrylate) (PBMA) membrane containing the $\left[\alpha, \alpha^{\prime}, \alpha^{\prime \prime}, \alpha^{\prime \prime \prime}\right.$-mesotetrakis(o-pivalamidophenyl)porphinato]cobalt(II)-1-methylimidazole (CoPIm) was prepared as in previous reports. ${ }^{8,9}$ Toluene solutions of the CoPIm complex and PBMA $\left(\bar{M}_{w}=320000\right)$ were mixed, and carefully cast on a Teflon plate under an atmosphere free of oxygen, then dried in vacuo, to yield a transparent membrane with a thickness of about $60 \mu \mathrm{m}$ and containing $2.5 \mathrm{wt} \% \mathrm{CoPIm}$.

\section{Spectroscopic Measurements}

Reversible oxygen-binding to the CoPIm complex fixed in the membrane was observed with spectral change in the visible absorption, using a high-sensitive spectrophotometer Shimadzu UV-2000 with a kinetic data processor. The spectrophotometer was equipped with a cell having a gas inlet and outlet tube. A membrane sample was stuck on the cell wall. A gaseous mixture of oxygen and nitrogen was introduced into the cell through the gas inlet and outlet tube with a standing pressure such that the total pressure in the cell came to be $1 \mathrm{~atm}$. Immediately after the gas was introduced into the cell, the time-course of the visible spectral change was monitored with a spectrophotometer.

\section{RESULTS AND DISCUSSION}

\section{Sorption Equilibrium}

The visible absorption spectrum of the red and transparent membranes is shown in Figure 1. The spectrum of the deoxy CoPIm complex $\left(\lambda_{\max }=528 \mathrm{~nm}\right)$ was changed to the spectrum with $\lambda_{\text {max }}=545 \mathrm{~nm}$ assigned to the oxy CoPIm

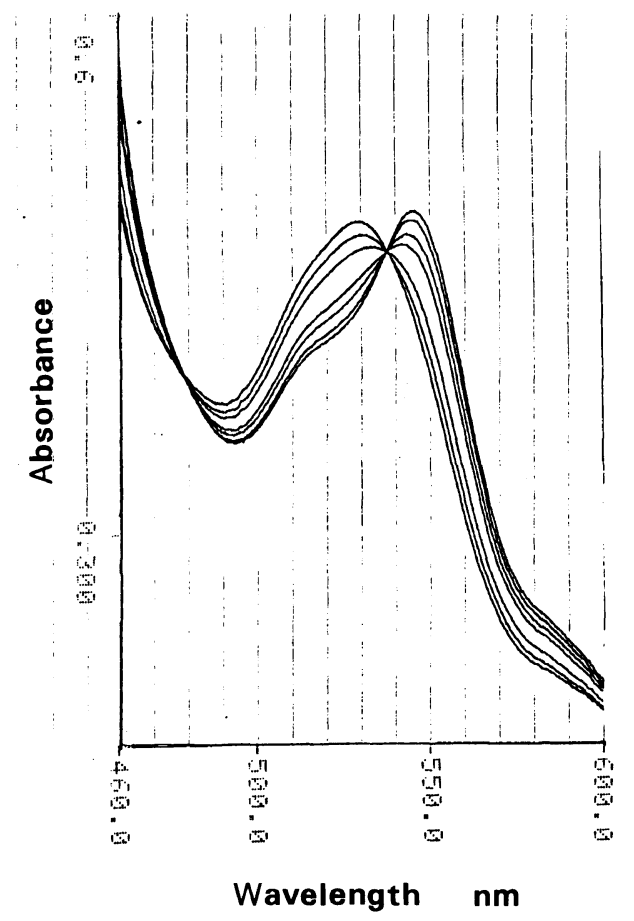

Figure 1. Visible absorption spectral change in the oxygen-binding to the CoPIm complex in the PBMA membrane (deoxy, $528 \mathrm{~nm}$; oxy, $545 \mathrm{~nm}$ ), at $25: \mathrm{C}$ $[\mathrm{CoPIm}]=2.5 \mathrm{wt} \%$.

complex $\left(\mathrm{O}_{2} / \mathrm{Co}=1 / 1\right.$ adduct, eq 1) immediately after exposure to oxygen. The oxydeoxy spectral change was reversible with isosbestic points at 480,538 , and $667 \mathrm{~nm}$. These visible absorption spectra agreed with those for the corresponding complex in toluene solution.

$$
\mathrm{CoPIm}+\mathrm{O}_{2} \rightleftharpoons \mathrm{CoPIm}-\mathrm{O}_{2}
$$

Sorption isotherms for molecular oxygen to the CoP complex in the membrane are shown in Figure 2, which were drawn by analyzing the spectral change of the membrane in response to the partial oxygen pressure in the cell using Drago's equation. ${ }^{10}$ The isotherms are the Langmuir types which means that the oxygen sorption in the membrane is interpreted in terms of the dual mode sorption model. The model postulates that the sorbed 


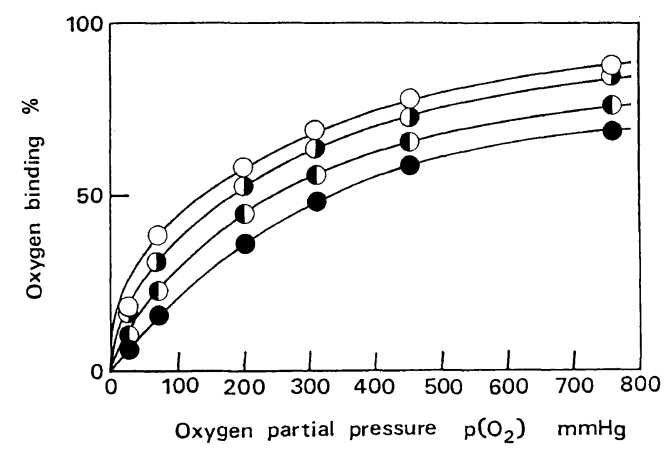

Figure 2. Sorption isotherms for oxygen to the CoPIm in the PBMA membrane, (at $\mathrm{O}, 20^{\circ} \mathrm{C} ; \mathrm{O}, 25^{\circ} \mathrm{C}$; $\left.30^{\circ} \mathrm{C} ; 0,35^{\circ} \mathrm{C}\right)[\mathrm{CoPIm}]=2.5 \mathrm{wt} \%$.

oxygen exists in two populations: one physically dissolved in the polymer matrix in a rubbery state according to Henry's law (its concentration $=C_{\mathrm{D}}$ ) and the other sorbed, in this case, to the CoP complex according to the Langmuir isotherm (its concentration $=C_{\mathrm{C}}$ ).

The dual sorption is represented by eq 2 , as has been described for the equilibrium sorption of gases in glassy polymers ${ }^{11}$ and in the polymer membrane containing adsorptive fillers. ${ }^{6}$

$$
C=C_{\mathrm{D}}+C_{\mathrm{C}}=k_{\mathrm{D}} p_{2}+C_{\mathrm{C}}^{\prime} K p_{2} /\left(1+K p_{2}\right)
$$

where $k_{\mathrm{D}}\left[\mathrm{cm}^{3}(\mathrm{STP}) \mathrm{cm}^{-3} \mathrm{cmHg}^{-1}\right]$ is the solubility coefficient for Henry's law, $C_{\mathrm{C}}^{\prime}$ is the saturated amount of oxygen reversibly sorbed to the binding site, in this case the CoP complex, $K$ is the oxygen-binding and -dissociation equilibrium constant to the complex, and $p_{2}$ is the upstream oxygen pressure.

The $k_{\mathrm{D}}$ values for the membrane were replaced by the ones for the PBMA membrane not containing the CoP complex. The $K$ values were determined by the oxygen-binding equilibrium curves in Figure 2. $k_{\mathrm{D}}$ and $K$ for the membrane are listed in Table I. In this study, $C_{\mathrm{C}}^{\prime}$ was directly determined to be 0.8 $\left[\mathrm{cm}^{3}(\mathrm{STP}) \mathrm{cm}^{-3}\right]$ from the concentration of the CoP complex. On the other hand, for the condensable penetrant sorption in glassy polymers, the Langmuir sorption capacity $\left(C_{\mathrm{H}}^{\prime}\right)$ is based on the frozen free volume in the polymers. The frozen free volume or "microvoid" arises from the non-equilibrium nature of the polymers in a glassy state, and a quantitative uncertainty remains in the $C_{\mathbf{H}}^{\prime}$ value. One of the advantages of the rubbery membrane containing the CoP complex as a fixed carrier is that $C_{\mathrm{H}}^{\prime}$, in eq 2 the $C_{\mathrm{C}}^{\prime}$ value, can be calculated from the CoP concentration in the membrane and that $C_{\mathrm{C}}^{\prime}$ is independent of temperature and upstream penetrant pressure.

\section{Sorption Kinetics}

For a Fickian diffusion, the sorption or desorption in an initial stage corresponds to diffusion in a semi-infinite medium: the diffusion equation, ${ }^{12}$ where a plot of $C(t) / C(\infty)$ vs. $t^{1 / 2} / l$ is linear, is given as follows

$$
C(t) / C(\infty)=\left(4 / \pi^{1 / 2}\right)\left(D t / l^{2}\right)^{1 / 2}
$$

and the diffusion coefficient is given as follows

$$
D=(\pi / 16) G^{2}
$$

where $C(t)$ is the cumulative amount of oxygen sorbed after $t(s), C(\infty)$ is the uptake amount at equilibrium, $l$ is the membrane thickness, and $G$ is the initial slope of the sorption or desorption curve. For the condensable penetrant sorption in glassy polymers at which the dual mode sorption model provides a description of the sorption equilibrium, Koros et al. have reported $^{4,13-15}$ that the sorption kinetic data are quite satisfactorily described by the Fickian kinetics if the interchange between species in the two sorption modes is rapid enough to establish local equilibrium. Although Fickian kinetics are precisely not suited for the dual mode sorption model, eq 3 and 4 were applied to kinetic data in this study to estimate dual mode transport parameters as have been done by Koros et al.

Time course of the oxygen sorption amount to the CoPIm complex (oxygen saturation degree of the complex $(\theta)$ ) in the membrane is able to be measured by the visible spectros- 


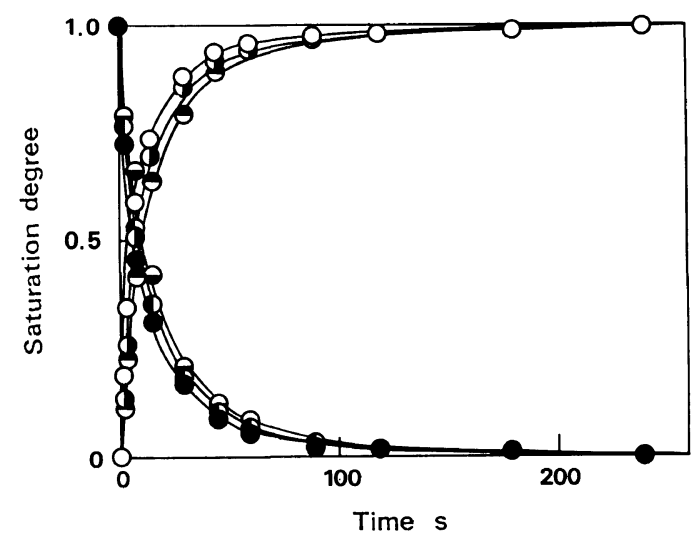

Figure 3. Time courses of the oxygen absorption and desorption in the CoPIm/PBMA membrane at $25^{\circ} \mathrm{C}$, (constant oxygen pressure $p_{2}$ at $\Theta$ and $\Theta, 70 \mathrm{mmHg}$; and $\bigcirc, 200 \mathrm{mmHg}$; $\bigcirc$ and $\bigcirc, 760 \mathrm{mmHg}$ ) [CoPIm] $=$ $2.5 \mathrm{wt} \%$.

copy after exposure of the membrane to a certain oxygen pressure $\left(p_{2}\right)$ as shown in Figure 3. Combination of the oxygen-binding $\%$ calculated from the $\theta$ value and the oxygenbinding equilibrium curve (Figure 2 ) yields the oxygen pressure $\left(p_{2}^{\prime}\right)$ to give the $\theta$ value. The product of this $p_{2}^{\prime}$ and $k_{\mathrm{D}}$ gives the oxygen sorption amount according to Henry's mode. The total sorption amount of oxygen is the sum of the oxygen sorption amount according to Henry's mode and that to Langmuir's mode. This treatment is based on the assumption that the equilibrium between the two sorption modes is attained fairly rapidly. Flash photolysis measurement on the oxygenbinding and -dissociation rate to and from the CoPIm complex in the membrane supports the validity of this assumption. ${ }^{9}$

Examples of the sorption kinetic runs are shown in Figure 4. The curves indicate that the sorptions and the transports of oxygen in the membrane satisfactorily fit the Fickian diffusion model. The diffusion coefficients increase with an increase in the upstream oxygen pressure (Figure 5), which suggests the occurrence of the dual mode transport of oxygen in the membrane containing the $\mathrm{CoP}$ complex, as has been often observed for condensable pen-

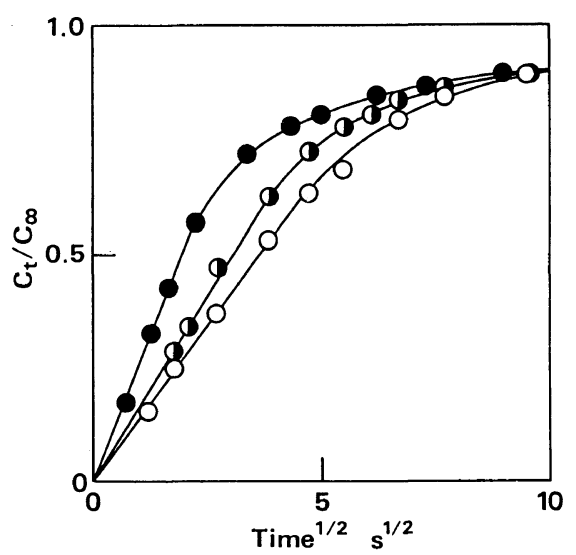

Figure 4. Sorption kinetic runs for the oxygen sorption in the $\mathrm{CoPIm} / \mathrm{PBMA}$ membrane at $25^{\circ} \mathrm{C}$, (constant oxygen pressure $p_{2}$ at $\bigcirc, 70 \mathrm{mmHg} ;$., $200 \mathrm{mmHg}$; $760 \mathrm{mmHg}$ ) $[\mathrm{CoPIm}]=2.5 \mathrm{wt} \%$.

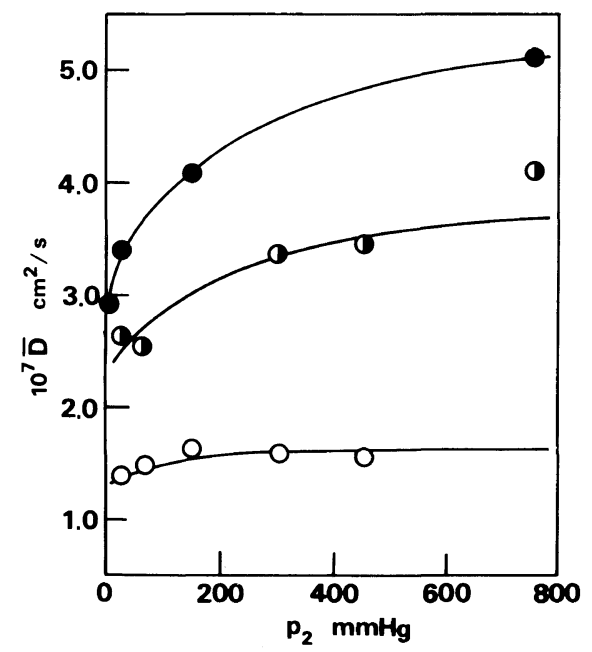

Figure 5. Effect of upstream oxygen pressure on diffusion coefficient for the CoPIm/PBMA membrane, (at $\left.\mathrm{O}, 25^{\circ} \mathrm{C} ; \mathbf{O}, 30^{\circ} \mathrm{C} ; 0,35^{\circ} \mathrm{C}\right)[\mathrm{CoPIm}]=2.5 \mathrm{wt} \%$.

etrant sorption in glassy polymers.

The dual mode transport model expresses the flux $(N)$ as $^{16}$

$$
N=-D_{\mathrm{D}}\left(\partial C_{\mathrm{D}} / \partial x\right)-D_{\mathrm{C}}\left(\partial C_{\mathrm{C}} / \partial x\right)
$$

where $D_{\mathrm{D}}$ and $D_{\mathrm{C}}$ are the diffusion coefficients for the Henry's $\left(C_{\mathrm{D}}\right)$ and the Langmuir's $\left(C_{\mathrm{C}}\right)$ populations respectively. The theoretical equation of the diffusion for the dual mode trans- 


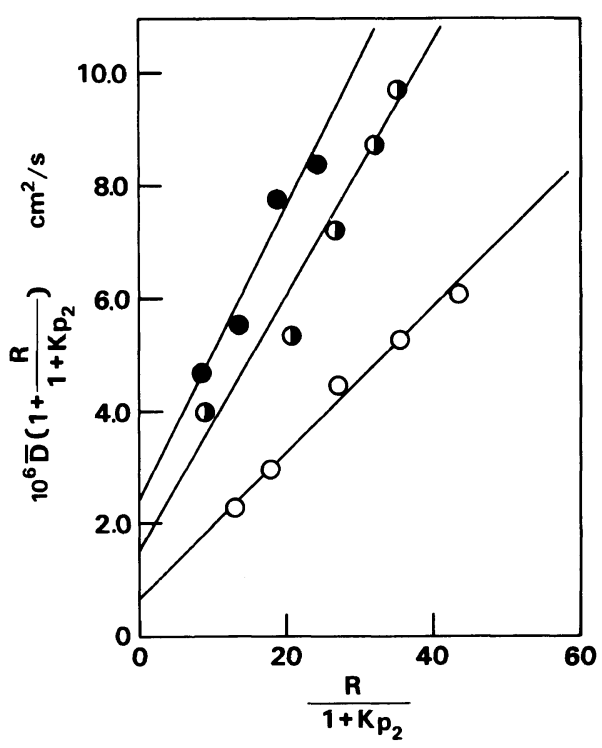

Figure 6. Analysis of diffusion coefficients in terms of eq 6 to determine $D_{\mathrm{C}}$ and $D_{\mathrm{D}}$ for oxygen in the CoPIm/ PBMA membrane, (at $\bigcirc, 25^{\circ} \mathrm{C} ; 0,30^{\circ} \mathrm{C} ; 0,35^{\circ} \mathrm{C}$ ) $[\mathrm{CoPIm}]=2.5 \mathrm{wt}^{\mathrm{o}} \%$.

port model is given as follow. ${ }^{4}$

$$
\begin{gathered}
\bar{D}=D_{\mathrm{D}}\left[\frac{1+F R /\left(1+K p_{2}\right)}{1+R /\left(1+K p_{2}\right)}\right] \\
F=D_{\mathrm{C}} / D_{\mathrm{D}}, \quad R=C_{\mathrm{C}}^{\prime} K / k_{\mathrm{D}}
\end{gathered}
$$

This equation indicates that a plot of $\bar{D}\left(1+R /\left(1+K p_{2}\right)\right) v s . K /\left(1+K p_{2}\right)$ produces a straight line with the slope $D_{\mathrm{C}}\left(=F D_{\mathrm{D}}\right)$ and the intercept $D_{\mathrm{D}}$. Figure 6 shows these plots for the oxygen sorption in the membrane containing a fixed carrier, using the $R$ and $K$ values taken from Table I and the diffusion coefficient from Figure 5. The plots show linear relationships, which support the dual mode transport of oxygen in the membrane and a pathway of oxygen transport via the CoP complex, i.e., the fixed carrier. The $D_{\mathrm{D}}$ and $D_{\mathrm{C}}$ values determined in this manner are listed in Table II. The results show that the Langmuir-sorbed penetrant has a mobility $c a .20 \%$ of that of the population based on the Henry's law. The $D_{\mathrm{D}}$ values in Table II almost agree with the $D_{\mathrm{D}}$ values determined from the permeability
Table I. Dual mode sorption parameters for oxygen

\begin{tabular}{|c|c|c|c|}
\hline Temp & $k_{\mathrm{D}} \times 10^{4}$ & $K \times 10^{2}$ & \\
\hline${ }^{\circ} \mathrm{C}$ & $\mathrm{cm}^{3}(\mathrm{STP}) \mathrm{cm}^{-3} \mathrm{cmHg}^{-1}$ & $\mathrm{cmHg}^{-1}$ & \\
\hline 20 & 9.94 & 8.70 & 72.4 \\
\hline 25 & 9.61 & 5.71 & 49.1 \\
\hline 30 & 9.30 & 4.02 & 35.7 \\
\hline 35 & 9.01 & 2.82 & 25.9 \\
\hline
\end{tabular}
to the CoPIm/PBMA membrane

Table II. Kinetic parameters for the oxygen sorption to the CoPIm/PBMA membrane

\begin{tabular}{ccccc}
\hline \multirow{2}{*}{ Temp } & $D_{\mathrm{D}} \times 10^{6}$ & & $D_{\mathrm{C}} \times 10^{7}$ & \\
${ }^{\circ} \mathrm{C}$ & $\mathrm{cm}^{2} \mathrm{~s}^{-1}$ & & $\mathrm{~cm}^{2} \mathrm{~s}^{-1}$ & \\
\hline 20 & 0.45 & 1.1 & 0.2 \\
25 & 0.65 & 1.3 & 0.2 \\
30 & 1.3 & 2.2 & 0.2 \\
35 & 2.3 & 2.6 & 0.1 \\
\hline
\end{tabular}

measurement for the CoP membrane $\left(7.0 \times 10^{-7}\right.$ and $1.1 \times 10^{-6} \mathrm{~cm}^{2} \mathrm{~s}^{-1}$ at 25 and $30^{\circ} \mathrm{C}$, respectively ${ }^{9}$ ).

Since the glassy polymer matrix undergoes plasticizing in the process of diffusive transport and $k_{\mathrm{D}}$ and $D_{\mathrm{D}}$ are not constant during a sorption run, application of the given equations for $D$ is not rigorously justified. ${ }^{4}$ On the other hand, oxygen sorption is more simply analyzed by the dual mode transport theory for our rubbery polymer membrane containing the complex as the fixed carrier of oxygen.

Acknowledgment. This work was partially supported by a Grant-in-Aid from the Ministry of Education, Science, and Culture of Japan.

\section{REFERENCES}

1. R. M. Barrer, J. A. Barrie, and J. G. Slater, J. Polym. Sci., 27, 315 (1958).

2. A. S. Michaels, W. R. Vieth, and J. A. Barrie, J. Appl. Phys., 34, 1 (1963).

3. W. R. Vieth, P. M. Tam, and A. S. Michaels, J. 
Colloid Interface Sci., 22, 360 (1966).

4. C. J. Patton, R. M. Felder, and W. J. Koros, J. Appl. Polym. Sci., 29, 1095 (1984).

5. Y. Kamiya, K. Mizoguchi, Y. Naito, and T. Hirose, J. Polym. Sci., Polym. Phys. Ed., 24, 535 (1986).

6. D. R. Paul and D. P. Kempt, J. Polym. Sci., Polym. Symp., 41, 79 (1973).

7. E. Tsuchida, J. Macromol. Sci., Chem., A13, 545 (1979).

8. H. Nishide, M. Ohyanagi, O. Okada, and E. Tsuchida, Macromolecules, 19, 495 (1986).

9. H. Nishide, M. Ohyanagi, O. Okada, and E. Tsuchida, Macromolecules, 20, 417 (1987).

10. T. Beugelsdijik and R. S. Drago, J. Am. Chem. Soc., 97, 6466 (1975).
11. W. R. Vieth, J. M. Howell, and J. H. Hsieh, J. Membrane Sci., 1, 177 (1976).

12. J. Comyn, Ed., "Polymer Permeability," Elsevier, New York, N. Y., 1985, p 26.

13. W. J. Koros, C. J. Patton, R. M. Felder, and S. J. Fincher, J. Polym. Sci., Polym. Phys. Ed., 18, 1485 (1980).

14. D. K. Yang, W. J. Koros, H. B. Hopfenberg, and V. T. Stannett, J. Appl. Polym. Sci., 30, 1035 (1985).

15. T. Uragami, H. B. Hopfenberg, W. J. Koros, D. K. Yang, V. T. Stannett, and R. T. Chern, J. Polym. Sci., Polym. Phys. Ed., 24, 779 (1986).

16. D. R. Paul and W. J. Koros, J. Polym. Sci., Polym. Phys. Ed., 14, 675 (1976). 